\title{
A Novel Dorsal Trimline Approach for Passive-Dynamic Ankle-Foot Orthoses
}

\author{
Hasan Kemal Surmen ${ }^{1}$ - Nazif Ekin Akalan² - Mahmut Cuneyt Fetvaci ${ }^{3}$ - Yunus Ziya Arslan ${ }^{3}$, \\ ${ }^{1}$ Istanbul University, Vocational School of Technical Sciences, Department of Automotive Technology, Turkey \\ 2 Istanbul Kültür University, Faculty of Health Sciences, Department of Physiotherapy and Rehabilitation, Turkey \\ 3 Istanbul University, Faculty of Engineering, Department of Mechanical Engineering, Turkey
}

\begin{abstract}
An ankle-foot orthosis (AFO) is an externally applied assistive device that encompasses the lower leg, ankle, and foot of the human body. In the current one-piece passive-dynamic AFO design, the trimming process is performed from lateral and medial parts of the ankle to ensure desired rotational displacement (hereafter referred to as Design I). In most cases, stress concentrations occurring over the trimmed regions during walking can cause permanent damage to the AFO. In this study, to reduce the stress concentration and ensure a homogeneous stress distribution, a new trimming approach is presented, in which the trim zones were transferred from lateral and medial to dorsal (hereafter referred to as Design II). Finite element analyses of the Designs I and II models were carried out. Displacement and von Mises stress values for both models under the same loading and boundary conditions were obtained. Maximum displacement values were $8.51 \mathrm{~mm}$ and $9.05 \mathrm{~mm}$ for Design I and Design II, respectively. Maximum stress values were $15.19 \mathrm{MPa}$ and 6.70 MPa for Design I and Design II, respectively. For the similar range of motion of ankle joint, the novel design produced less stress and more homogeneous stress distribution than the currently used design, thus indicating that Design II would be more resistant to plastic deformation than Design I.
\end{abstract}

Keywords: dorsal trimline, ankle-foot orthosis, finite element analysis, computer-aided design, 3D optical scanning

Highlights

- A new trimming approach has been developed for ankle-foot-orthoses (AFOs).

- The new method reduced the occurrence of the lateral cracks seen in the one-piece passive-dynamic AFOs during walking.

- Similar displacement values were obtained with fewer stresses by transferring the trimming zone from lateral/medial sides to the dorsal side.

- With the new trim approach, the stresses distributed more homogeneously over the AFO, while the maximum stress was reduced by more than half.

- These results encourage researching the use of this novel trim approach in AFOs produced for many different biomechanical purposes.

\section{INTRODUCTION}

Ankle-foot orthosis (AFO) is a specially designed supportive medical device which is intended to i) control the motion of the ankle and subtalar joints, ii) compensate for muscle weakness and drop foot, iii) correct deformities in the ankle and subtalar joints due to injuries [1] and [2], stroke [3] and [4], and congenital anomalies such as cerebral palsy [5] to [7] and spina bifida [8] and [9]. Many different types of AFO are available for different biomechanical and clinical purposes. They can generally be classified into two categories as articulated [10] and [11] and nonarticulated [12] and [13]. Articulated AFOs contain mechanical joints at the ankle section that allow or limit the range of ankle motion in the sagittal plane. Similarly, non-articulated, passive AFOs are used to control the ankle movement, but they are constructed as one piece without the incorporation of a joint mechanism.

AFOs are exposed to external loadings from different directions during dynamic tasks, such as standing, swaying, or walking. Unlike from a solid AFO, which is typically stiff and rigid, a dynamic AFO (provides subtalar stabilization while allowing free or restricted dorsiflexion and plantarflexion) is characteristically thin, flexible and wraps around the patient's entire foot and ankle in order to provide improved sensation and alignment [14]. Due to the exposure to repetitive and excessive loadings, which create a high level of stresses concentrated over the ankle part of the AFO, dynamic AFOs undergo plastic deformation and hence, in time, they cannot satisfactorily fulfil movement restriction, which means that the patients cannot effectively benefit from the AFO [15].

Design parameters, such as material type, material thickness, and trimline geometry, influence the stiffness of the AFO, which is determined according to the biomechanical needs of the patients. Non-articulated AFOs have a rigid character if the trimlines are anterior to the malleoli. As trimlines are moved posterior to the malleoli, the AFO's flexibility is enhanced [16]. The range of motion of an orthosis 
around the ankle joint is mainly specified by the type of the trimline. In the design stage of the trimline, in addition to the determination of the range of motion, prevention of the plastic deformation should also be taken into consideration. The plastic deformation frequently occurring in the orthosis along the trimline emerges as a result of the permanent damage, which decreases the supportive feature of the orthosis. Therefore, the challenge in the design process of an $\mathrm{AFO}$ is to be able to provide a desired range of motion with minimal stress concentration over the material.

Bielby et al. [17] designed three different trimlines (conservative, moderate, aggressive approaches) in the medial and lateral sections of the orthosis at different depths and compared the stiffness of the AFOs. The models with different trimline depths resulted in significantly different stiffness measures, except for the moderate and aggressive cuts along the transverse plane. This study verified that the trimming more material would lead to less stiff and more extensible specimens. Sumiya et al. [18] observed the rigidity of the orthoses according to the different trimlines formed in the ankle part of the AFO. They claimed that the orthotic stiffness could be adjusted by trimming to exactly meet the individual requirements. In these experimental studies, no computational approach such as finite element technique was used and hence stress distribution over the orthosis was not observed. Chu et al. [19] performed a three-dimensional finite element analysis (FEA) of an AFO model and identified the areas where the stresses were maximum on the AFO. The AFO model they analysed in their studies was obtained using a computer-aided design (CAD) program. However, the proposed AFO was far from the real anatomical structure because the number of surfaces comprising the model was very low.

The design variables should be carefully evaluated to reach the optimal design. At this point, FEA provides an opportunity for designers to predict the behaviours of their product under different loading and boundary conditions. Therefore, alternative designs can be tested for their validity and safety using the computational techniques before any model is built [20]. FEA reduces the cost and time needed to develop the optimal AFO that requires design alterations. However, the solid body model used in the finite element approach should be properly constructed such that it accurately represents the mechanical structure of the original product; thus, it would be possible to have realistic results from the analysis. Therefore, the model geometry, boundary conditions, material type, loading conditions, and meshing preferences, which are the major components of the FEA, should be defined as accurately as possible to be able to mimic real-world conditions.

The aim of this work was to establish a novel trimline design to avoid permanent deformations, which can occur in plastic AFOs exposed to various loads during walking, by homogenizing the stress distributions and reducing the magnitude of the stresses over AFO. First, the mechanical analysis of a one-piece passive-dynamic AFO model trimmed from medial and lateral parts (hereafter referred to as Design I) was performed by using the finite element method and the theoretical results were compared with experimental data to check whether the simulation provided reliable results. Then, once the verification of the finite element solution of the AFO has been done, a new finite element model was developed and mechanically analysed for the AFO trimmed with a novel trimline approach, in which the trim zones were transferred from lateral and medial sides to the dorsal side (hereafter referred to as Design II). Finally, displacement and stress values obtained from the FEAs of the currently used and novel models were evaluated comparatively.

\section{METHODS}

\subsection{AFO Production}

The currently used AFO, which was experimentally evaluated in this study, was fabricated from a polypropylene sheet with $3 \mathrm{~mm}$ thickness using the vacuum moulding technique [21] by an experienced orthotic specialist who has worked more than 10 years with neurologically affected patients. In this technique, the polypropylene sheet was heated to its softening temperature in an oven. A positive mould was then surrounded by the polypropylene sheet, thereby producing a negative mould. In the study, moulds were produced according to the lower limb of a patient (age: 12, height: $161 \mathrm{~cm}$, mass: $60 \mathrm{~kg}$ ) with spina bifida, which is a neurological disease stemming from a spinal cord malformation and might cause physical and intellectual disabilities that range from mild to severe. The patient did not use any additional walking aids and had no knee and hip flexion or ankle dorsiflexion contracture. The purpose of the use of the AFO was to reduce the increased dorsiflexion and knee-hip flexion in the stance phase. To regain a natural gait pattern, AFO should ideally allow dorsiflexion not higher than $12^{\circ}$ in the middistance of the gait cycle. The AFO was first trimmed so that the proximal trimline was horizontal and $2 \mathrm{~cm}$ below the fibula head, passed $1 \mathrm{~cm}$ anterior to the tip 
of the malleoli, and the metatarsal trimline was just posterior to the metatarsal heads. By doing so, a rigid (solid) AFO was obtained (Fig. 1a). Then, the AFO was trimmed beyond the trimline of the solid AFO such that it allowed flexibility under gait conditions (dynamic AFO) (Fig. 1b).
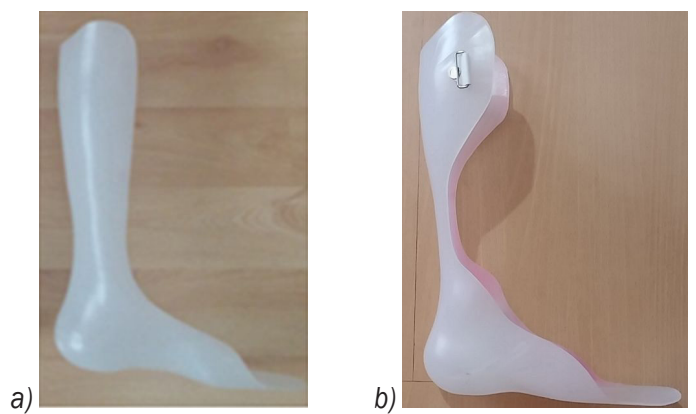

Fig. 1. a) Rigid and b) flexible (dynamic) AFO used in the study

\subsection{Three-Dimensional CAD Model of the AFO}

Topometric three-dimensional (3D) scanning systems are generally used to measure the dimensions of devices or to convert the physical objects into 3D data [22] and [23]. AFOs have free-form geometry. Thus, it is difficult to draw a 3D AFO model with a CAD program. However, scanning technology

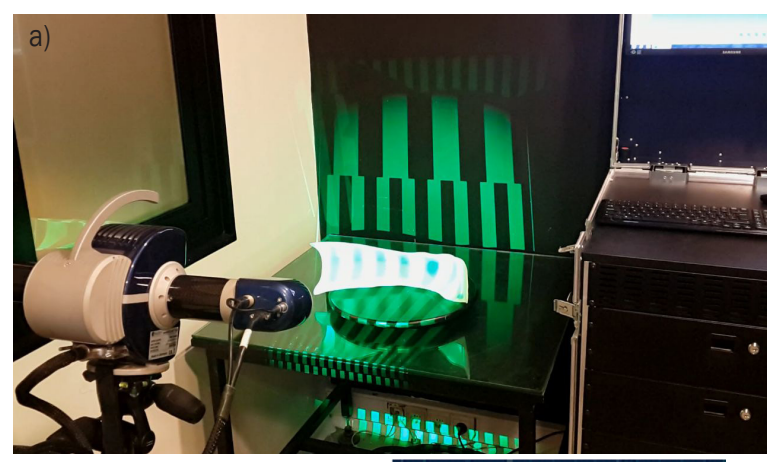

b)

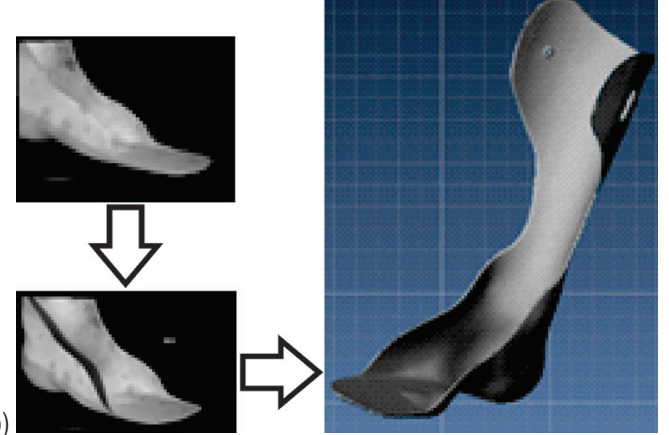

Fig. 2. a) Optical scanning of $A F O$, b) conversion of the physical model of the AFO to a CAD model provides a complete 3D AFO model for analysing in computational simulation software. An optical 3D scanner (Breuckmann $\mathrm{GmbH}$ ) based on the fringe projection system was used to digitize the AFO model with Design I (Fig. 2a). It is possible to obtain highly precise $3 \mathrm{D}$ data with this sophisticated method of high-resolution 3D digitization [24] and [25]. 20 scanned images were combined, and a single point cloud model was obtained. Then the model was converted into a 3D solid body model (Fig. 2b). In this process, 4000 surfaces were used to represent the real model as well as possible in regard to the geometric details. The conversion process takes time in proportion to the number of surfaces used. Additionally, deviation analysis was carried out for the $3 \mathrm{D}$ model with a tolerance of $0.1 \mathrm{~mm}$. It was ensured that all dimensions of the model were below the pre-determined tolerance value.

\subsection{Experimental Measurements}

The objective of the experiment was to measure the displacements between two points along the anteroposterior axis, i.e. the force application point and a predefined origin (stable) point of global coordinate axis under various loading conditions (Fig. 3). AFO was fixed to a stable structure from its footplate and heel by clamps. Two weight carriers were hung from the rings which were located at the medial and lateral sides of the AFO.

In the experiments, displacement values were measured using a digital calliper for four different force values. Each carrier applied a force of 2 N. Later, forces of $10 \mathrm{~N}, 20 \mathrm{~N}$, and $30 \mathrm{~N}$ were successively placed on the carrier (Fig. 4). Consequently, forces of $4 \mathrm{~N}, 14 \mathrm{~N}, 24 \mathrm{~N}$, and $34 \mathrm{~N}$ were applied to the AFO.

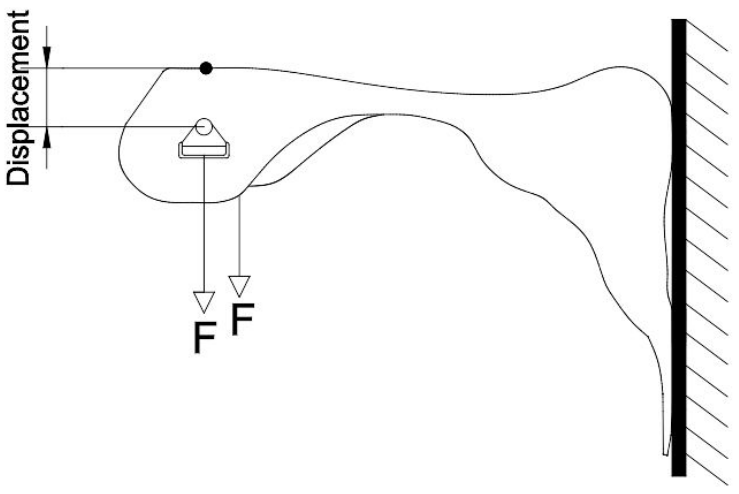

Fig. 3. Sketch of the experimental setup; by using this setup, displacements between the force (F) application point and a predefined origin point were measured 

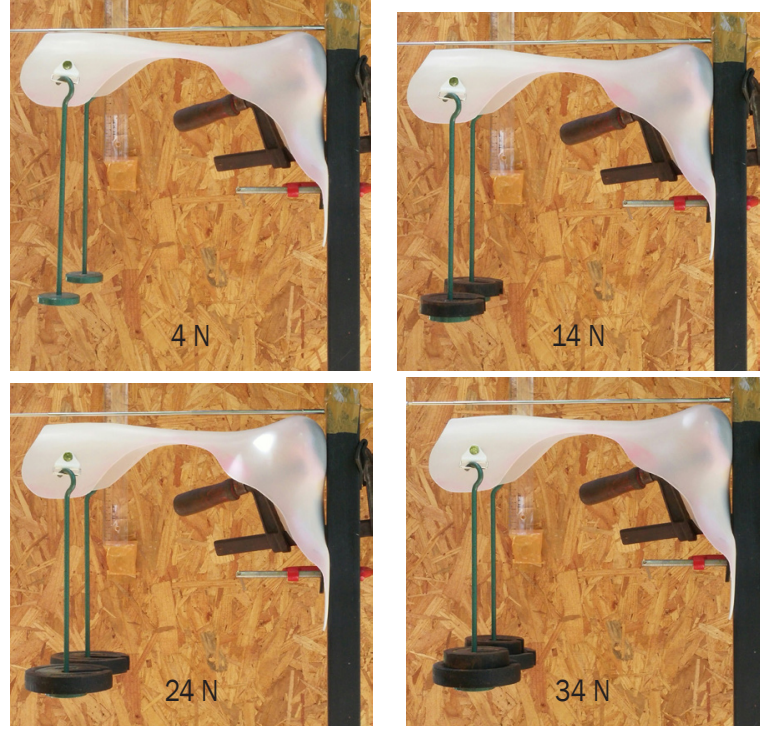

Fig. 4. Experimental setup showing the various loading combinations subjected to the AFO

\subsection{Finite Element Analysis of the AFO Model}

To analyse the mechanical behaviour of the AFO with Design I exposed to various loadings using FEA, a digitized 3D AFO model was imported in the CAD software (SolidWorks Corporation, Concord, MA, USA). In the software, planes of the model were arranged according to global $x, y, z$ directions. Polypropylene copolymer material was chosen from the software library and defined as linear, isotropic, and elastic material. The mechanical properties of the material are given in Table 1.

Table 1. Mechanical properties of the AFO material used in the study

\begin{tabular}{cc}
\hline Tensile strength & $27.6 \mathrm{MPa}$ \\
\hline Elastic modulus & $896 \mathrm{MPa}$ \\
\hline Poisson's ratio & 0.4103 \\
\hline Mass density & $890 \mathrm{~kg} / \mathrm{m}^{3}$ \\
\hline Tearing modulus & $315.8 \mathrm{MPa}$ \\
\hline
\end{tabular}

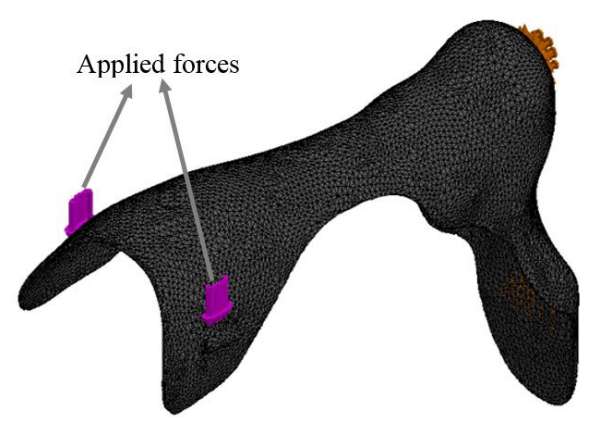

Fig. 5. Finite element model of the AFO
As for the boundary conditions, the model was fixed from its footplate and heel surfaces which were also immobilized during the experiments by means of fixed joints. Forces were applied from each side where the strap was linked to the AFO (Fig. 5). Mesh size was determined around $4 \mathrm{~mm}$ considering the detailed surface of the model and a high-quality mesh structure was generated using solid mesh type. The mesh structure consisted of 96883 nodes and 48803 elements.

The experimental and theoretical displacement results are shown in Table 1. Experimentally, forces of $4 \mathrm{~N}, 14 \mathrm{~N}, 24 \mathrm{~N}$, and $34 \mathrm{~N}$ resulted in displacement values of $2.28 \mathrm{~mm}, 7.61 \mathrm{~mm}, 13.95 \mathrm{~mm}$, and 18.5 $\mathrm{mm}$ along the anteroposterior axis, respectively. Theoretical displacement values obtained from FEA were $2.14 \mathrm{~mm}, 7.86 \mathrm{~mm}, 13.65 \mathrm{~mm}$, and $18.73 \mathrm{~mm}$ for the same loading conditions as the experimental ones. We calculated the absolute percentage error (e) between the experimental (disp exp) and theoretical displacement $\left(\right.$ disp $\left._{\text {theo }}\right)$ values as seen below.

$$
e=100 \cdot \frac{\mid \operatorname{disp}_{\exp }-\text { disp }_{\text {theo }} \mid}{\operatorname{disp}_{\exp }} .
$$

It can be seen from the table that all percentage errors between the displacements obtained from the experimental and FEA processes were below the rate of $6.1 \%$. It can be deduced from the levels of percentage errors that the displacement values of FEA are in good agreement with the experimental results.

To demonstrate the locations in which the maximum stress values occurred over the AFO model, a representation of the stress distribution is given in Fig. 6. It can be observed from the figure that maximum stress values over the Design I AFO model occurred around the medial and lateral sides.

The agreement between the displacement values obtained from the experiment and FEA of the AFO model indicated that the finite element modelling approach could be used for the mechanical characterization of new AFO models.

\subsection{The Novel Trimline Approach for the AFO}

In the second part of the study, a new trimline approach was developed to reduce the stress concentration on the anterior side of the ankle of the AFO, thereby avoiding permanent deformations and ensuring the proper functioning of the AFO. The similar procedures applied in the first part of the study, namely i) fabrication of the rigid AFO using the vacuum moulding technique, ii) digitization of the dimensions of the AFO using the 3D scanning 
Table 2. Externally applied forces, and experimental and theoretical displacement values along the anteroposterior axis

\begin{tabular}{ccccccc}
\hline \multicolumn{2}{c}{ Applied Forces [N] } & \multicolumn{3}{c}{ Displacement [mm] } & $\begin{array}{c}\text { Difference between the experimental and } \\
\text { theoretical displacements [mm] }\end{array}$ & Percentage error [\%] \\
\cline { 1 - 4 } Medial side & Lateral side & Total & Experiment & Finite element analysis & 0.14 & 6.1 \\
\hline 2 & 2 & 4 & 2.28 & 2.14 & 0.25 & 3.2 \\
\hline 7 & 7 & 14 & 7.61 & 7.86 & 0.30 & 2.1 \\
\hline 12 & 12 & 24 & 13.95 & 13.65 & 0.23 & 1.2 \\
\hline 17 & 17 & 34 & 18.5 & 18.73 & Mean & 3.15 \\
\hline
\end{tabular}

a)

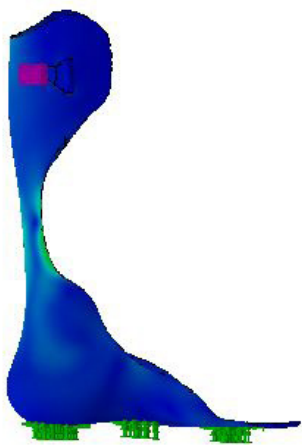

b)

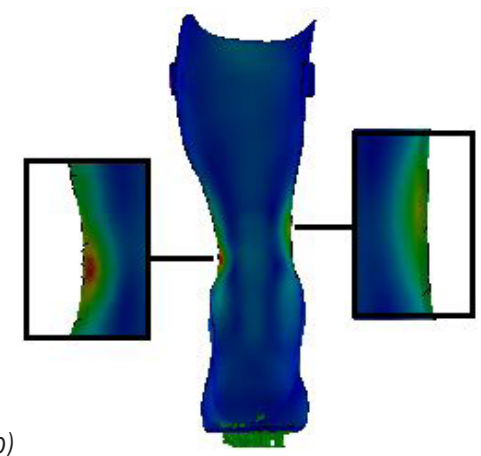

c)

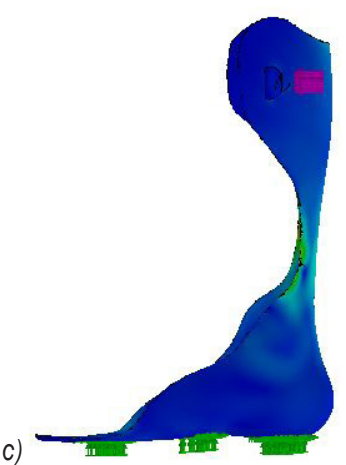

Fig. 6. Stress distribution over the AFO model: views a) from lateral, b) frontal, and c) medial planes

system and obtaining the single point cloud model, and iii) conversion of the cloud model to a $3 \mathrm{D}$ solid body model, were applied to obtain another solid AFO model in the second part.

Two different AFO models were created from the CAD model of solid AFO. The first model, which was trimmed from the lateral and medial parts of the AFO, represents the traditional AFO (Fig. 7a) while the second model, which was trimmed from the dorsal part, represents the novel trimline approach (Fig. 7b).

a)

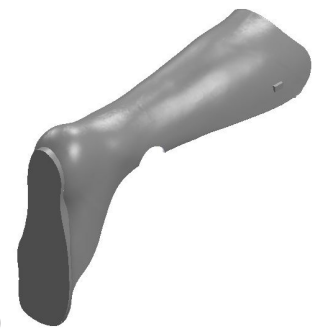

b)

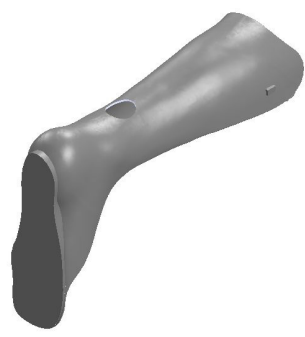

Fig. 7. a) The AFO with Design I trimmed from medial and lateral sides; b) the new AFO (Design II) trimmed from dorsal side

Trimming was done around the ankle, where the AFO was most exposed to deformation. In the AFO model with Design I (Fig. 7a), two half-circle pieces with a diameter of $36 \mathrm{~mm}$ were cut out from both lateral and medial sides of the orthosis. In the novel model with Design II (Fig. 7b), the same cross-sectional area, as in the traditional model, was extracted from the dorsal side. Considering that the orthosis will be worn with shoes, the footplate of the new model was modelled according to the shoe soles. This model also allowed the fixing process to be done by selecting only a single surface in the FEA.

In both finite element models, a total of $34 \mathrm{~N}$ forces, $17 \mathrm{~N}$ from the medial superior and lateral superior to the ankle each, were applied in the anteroposterior direction (Fig. 8). Both models were meshed using parabolic tetrahedral elements and were supported by fixed joints from the dorsal side of the footplates. The mesh structure consisted of 102725 nodes, 52487 elements for the model with Design I and (Fig. 8a) and 103507 nodes, 53005 elements for the novel design (Fig. 8b). Since it is widely used in the moulded plastic AFOs, polypropylene was determined as the orthosis material, and the finite element solution was performed in the linear region.

\section{RESULTS}

After the completion of the FEA, the displacement (Fig. 9) and von Mises stress (Fig. 10) values for Design I and Design II models under the same loading and boundary conditions along the anteroposterior axis were obtained. Maximum displacement values, which occurred at the medial and lateral sides of the 
a)

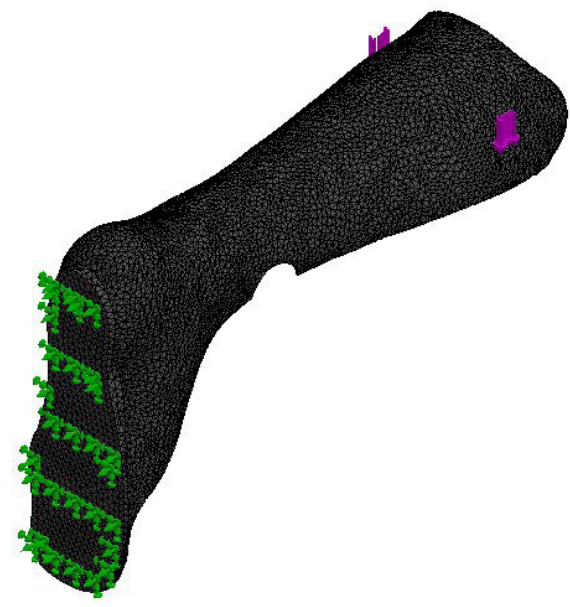

b)

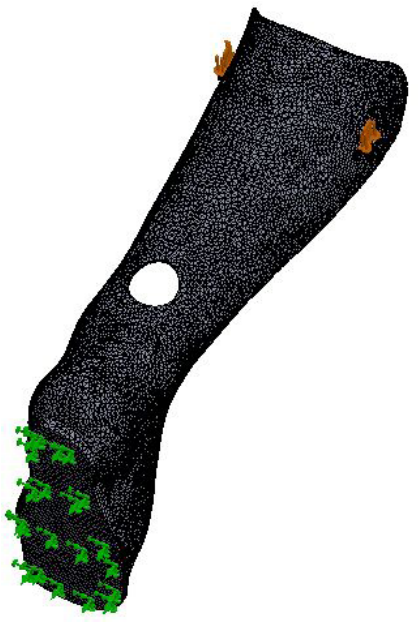

Fig. 8. Finite element models of the a) currently used, and b) newly designed AFOs

a)

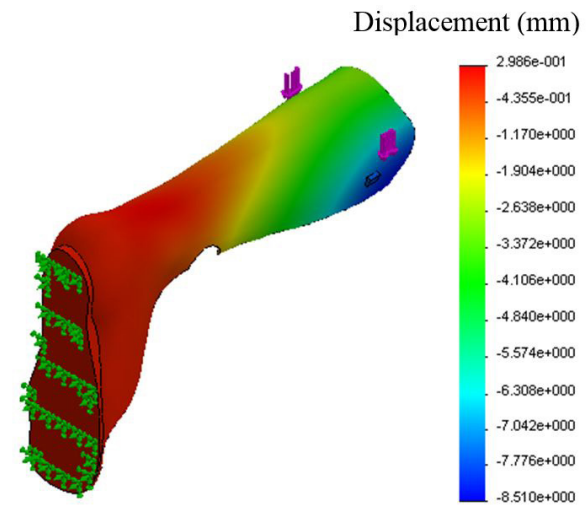

b)

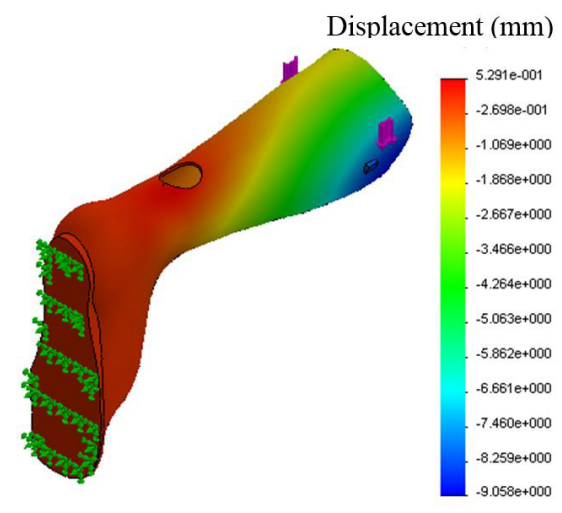

Fig. 9. Displacement values obtained from a) Design I and b) Design II AFOs under the same loading and boundary conditions

a)

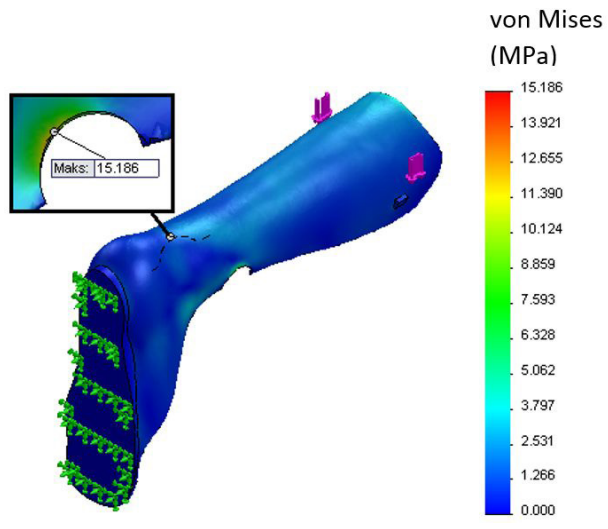

b)

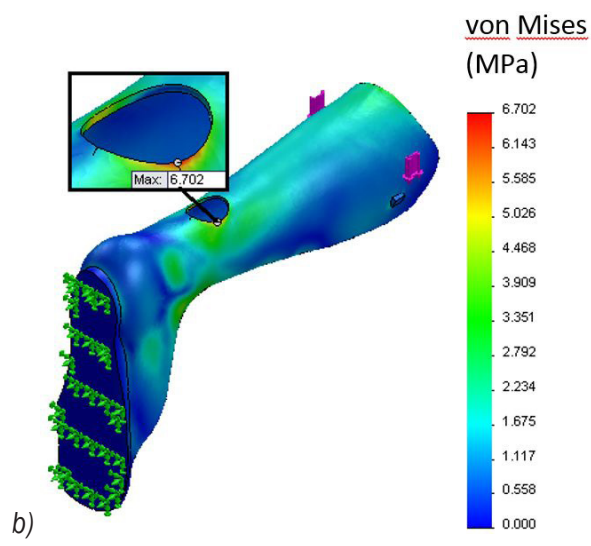

Fig. 10. von Mises stress values obtained from a) Design I, and b) Design II models under the same loading and boundary conditions

ankle, were $8.51 \mathrm{~mm}$ (Fig. 9a) and $9.05 \mathrm{~mm}$ (Fig. 9b) for Design I and Design II models, respectively.

Maximum von Mises stress values, which occurred at the edges of the trimlines, were $15.19 \mathrm{MPa}$ (Fig. 10a) and 6.70 MPa (Fig. 10b) for the Design I and Design II models, respectively.
Figs. 11 and 12 show the von Mises stress values of some points along the bending line around the ankle of the AFO. As can be seen in Fig. 11, a significant amount of the stresses was concentrated in the medial and lateral sections, and the maximum tension is obtained in the medial section as $15.19 \mathrm{MPa}$. 


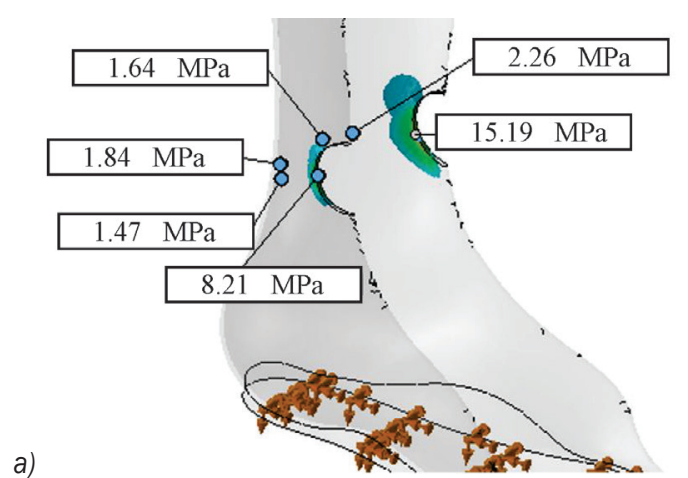

b)

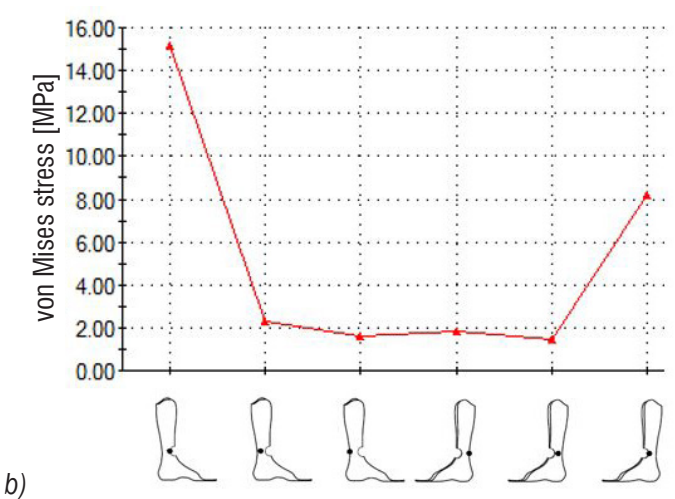

Fig. 11. a) Von Mises stress values of some nodes from medial to lateral along the bending side of the classically trimmed AFO; b) the graphical representation of the corresponding stress values from lateral to medial part
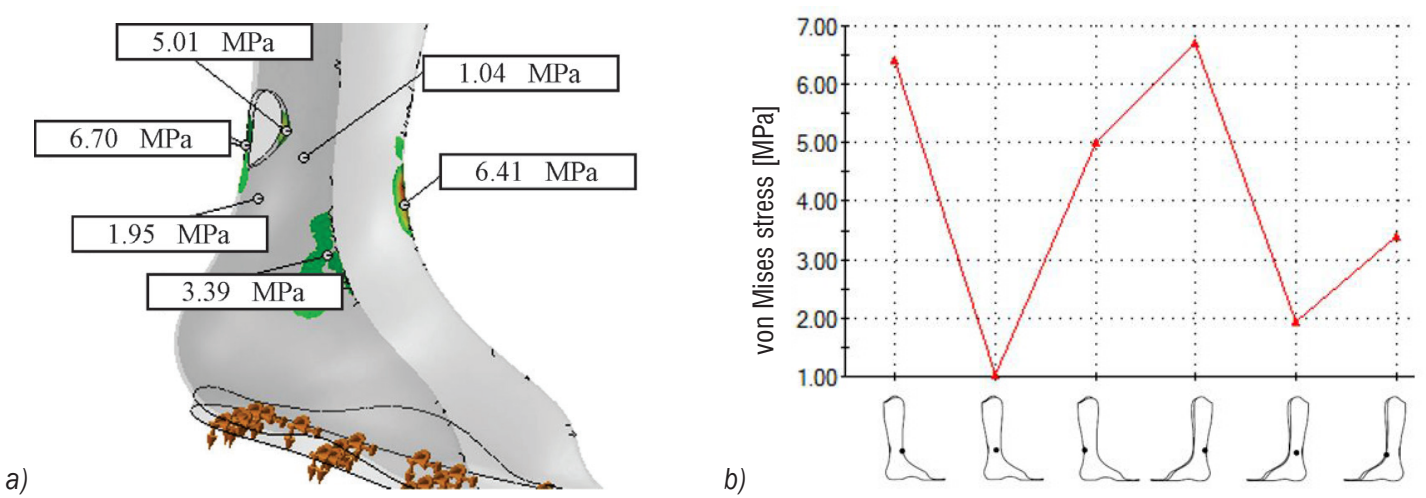

Fig. 12. a) von Mises stress values of some nodes from medial to lateral along the bending side of the novel AFO; b) the graphical representation of the corresponding stress values from lateral to medial part

In the new AFO model, which was designed to distribute the stresses more homogeneously over the $\mathrm{AFO}$, some of the stresses were transferred to the trim zone formed in the dorsal region (Fig. 12), thus avoiding high stresses concentrated in the medial and lateral parts.

\section{DISCUSSION}

AFO is used to control instabilities of the lower limb by compensating for the muscle weakness and aligning the positions of ankle and foot properly. Traditional plastic AFOs are widely prescribed due to their properties of meeting a variety of biomechanical needs, be easily produced and relatively low cost. In AFO design, ankle stiffness, which is an important parameter that determines the major mechanical characteristics of the AFO, depends on different structural properties such as trimline, material type, and material thickness. The effects of these parameters can be practically tested by using the finite element method. By doing so, effects of the design modifications on the mechanical properties of the AFO can be investigated using FEA without conducting experimental testing which would result in high costs. However, to be able to obtain accurate and reliable results from such computational techniques, one should take into account the patient factors in addition to the mechanical properties of the AFO, which would directly affect the mechanical behaviour of the AFO, such as the dynamic forces created during gait transmitted from contact points to the AFO, level of the activity and severity of the disorder of the patient, walking speed, and terrain where patient is walking.

The most preferred and easiest way to modify the stiffness of an AFO is to trim the lateral and medial parts of it. However, trimming of these parts can lead to stress concentration around these regions and thereby, as it is seen in orthosis clinics, resulting in plastic deformations and cracks on the AFO.

In this study, we concentrated on reducing the high stress values, which occur in the currently used AFOs and lead to permanent deformations, by using 
a new trimline approach. With this approach, the trimline region was transferred from the medial and lateral sides of the AFO to the dorsal side, resulting in a more homogeneous stress distribution (Fig. 11b, Fig. $12 b)$. When the mechanical results obtained from the currently used and novel techniques were compared, it was seen that fewer stress values were obtained from the novel model than that of the Design I model, while the displacement values obtained from both models were close to each other. For the similar range of motion of ankle joint, Design II produced fewer stress values than Design I, thus indicating that the novel design with dorsal trimline would be more resistant to plastic deformation than the currently used design with medial and lateral trimlines would. With the use of the novel model, the maximum stress value seen in the Design II model was reduced by more than half. The results derived from our study can be employed to make different conceptual AFO designs for different biomechanical requirements.

A three-dimensional solid body model of the AFO is required for FEA. However, it is difficult to draw such an object with complex and free-form geometry with CAD software [26]. On the other hand, with the advances in the scanning technology, 3D CAD model of an object with physical free-form geometry can be obtained, thereby enabling the object to be mechanically analysed with finite element software. In our study, AFO was converted into the high-quality point cloud data and a 3D solid object by a 3D optical scanning system and some commercial software packages, respectively. Obtaining a 3D AFO model with high-resolution would play a crucial role in obtaining reliable mechanical results from the FEA.

Gomes et al. evaluated the effect of structural reinforcements, which are applied to AFOs to prevent the lateral cracks that are occurring over AFOs, by using FEA [27]. They tried to solve a common problem, which is the opening of cracks on the lateral sides of AFOs due to the stress forces applied during the gait cycle, by using some reinforcement methods. Munguia and Dalgarno demonstrated AFO optimization by means of composite reinforcement [28]. They studied the possibilities of generating modular AFO elements using additive manufacturing techniques, and they suggested an alternative way for the design and manufacture of orthoses and reinforcement approach by using $3 \mathrm{D}$ printing technology. We think that the new trim approach could eliminate the need for reinforcement procedures to increase the strength of AFOs, thereby enabling to save time and cost.
In the scope of the study, analyses were carried out in the linear region, and the material was assumed to have an elastic property, which can be considered one of the limitations of the study. The study can be developed by performing viscoelastic analysis in which higher forces that lead to plastic deformation are applied. Furthermore, in the currently used trimming process made from the lateral and medial parts of the $\mathrm{AFO}$, it is easy to work with cutting apparatus and the process can be done in a short time [29]. In the proposed dorsal trimming method, trimming tasks can be more difficult and time-consuming. In this study, we carried out the static analysis. However, fatigue analysis including dynamic loading conditions would also provide a comprehensive insight regarding the mechanical responses of the AFO with different designs. The trimlines used in the study were determined to have uniform circular geometries to accurately compare two different AFO models. However, in the conventional AFOs used in practice, trimlines do not show a proper geometric feature. The accurate results regarding the performance of the novel AFO in terms of the comfort, metabolic energy consumption, ergonomics, and the correction of the deformities would be obtained from the clinical trials which will be the next step of our ongoing research.

\section{CONCLUSIONS}

In this study, a new trimming approach has been developed to reduce the occurrence of the lateral cracks seen in the one-piece passive-dynamic AFOs during walking. A dorsal trim method was applied to diminish the stress concentrations in certain regions of the AFO. The results showed that similar displacement values were obtained with fewer stresses by transferring the trimming zone from the lateral/medial sides to the dorsal side. With the new trim approach, the stresses distributed more homogeneously over the AFO, while the maximum stress was reduced by more than half. These results encourage researching the use of this novel trim approach in AFOs produced for many different biomechanical purposes. In clinical trials, the appropriateness of the use of AFOs produced by this new trim method could be investigated, and merits and shortcomings of the new design can be practically evaluated.

\section{ACKNOWLEDGEMENTS}

This research was supported by The Research Fund of the Istanbul University, Project No. BEK-2017-25426. 


\section{REFERENCES}

[1] Emmens, A., Pisotta I., Masciullo, M., van Asseldonk, E., van der Kooji, H. (2017). Improving the standing balance of people with spinal cord injury through the use of a powered ankle-foot orthosis. González-Vargas, J., Ibá-ez, J., Contreras-Vidal, J., van der Kooij, H., Pons, J. (eds.), Wearable Robotics: Challenges and Trends, Biosystems \& Biorobotics, Springer, Cham, vol. 16, p. 415-419, D0l:10.1007/978-3-319-46532-6_68.

[2] Brown, S.E., Esposito, E.R., Wilken, J.M. (2017). The effect of ankle foot orthosis alignment on walking in individuals treated for traumatic lower extremity injuries. Journal of Biomechanics, vol. 61, no. 1, p. 51-57, D0l:10.1016/J.jbiomech.2017.06.037.

[3] Kesikburun, S., Yavuz, F., Güzelküçük, Ü., Yaşar, E., Balaban, B. (2017). Effect of ankle foot orthosis on gait parameters and functional ambulation in patients with stroke. Turkish Journal of Physical Medicine and Rehabilitation, vol. 63, no. 2, p. 143148, DOI:10.5606/tftrd.2017.129.

[4] Tyson, S.F., Kent, R.M. (2013). Effects of an ankle-foot orthosis on balance and walking after stroke: a systematic review and pooled meta-analysis. Archives of Physical Medicine and Rehabilitation, vol. 94, no. 7, p. 1377-1385, D0l:10.1016/j. apmr.2012.12.025.

[5] Pauk, J., Innatouski, M., Daunoraviciene, K., Laskhousky, U., Griskevicius, J. (2016). Research of the spatial-temporal gait parameters and pressure characteristic in spastic diplegia children. Acta of Bioengineering and Biomechanics, vol. 18, no. 2, p. 121-129, D0I:10.5277/ABB-00393-2015-02.

[6] Meyns, P., Kerkum, Y., Buizer, A., Becher, J., Brehm, M., Harlaar, J. (2016). The effect of ankle foot orthosis stiffness on trunk movement and walking energy cost in cerebral palsy. Gait \& Posture, vol. 49, no. 2, p. 2, D0l:10.1016/j. gaitpost.2016.07.070.

[7] Sabbagh, D., Fior, J., Gentz, R. (2016). Long-term effects of a dynamic ankle foot orthosis on a patient with cerebral palsy following ischaemic perinatal stroke-a case study. Gait \& Posture, vol. 49, no. 2, p. 224, D0l:10.1016/j. gaitpost.2016.07.277.

[8] Ivanyi, B., Schoenmakers, M., van Veen, N., Maathuis, K., Nollet, F., Nederhand, M. (2015). The effects of orthoses, footwear, and walking aids on the walking ability of children and adolescents with spina bifida: a systematic review using International classification of functioning, disability and health for children and youth (IC-CY) as a reference framework. Prosthetics and Orthotics International, vol. 39, no. 6, p. 437443, DOI:10.1177/0309364614543550.

[9] Abelheira, L.A., Ribeiro, M.G, Salomão J.F.M, Orsini M. (2016). Benefits from using an ankle-foot orthosis in children with myelomeningocele. Revista Brasileira de Neurologia, vol. 52, no. 1, p. 30-34.

[10] Kobayasi, T., Orendurff, M.S., Hunt, G., Lincoln, L.S., Gao, F., LeCursi, N., Foreman, K.B. (2017). An articulated ankle-foot orthosis with adjustable plantarflexion resistance, dorsiflexion resistance and alignment: a pilot study on mechanical properties and effects on stroke hemiparetic gait. Medical Engineering \& Physics, vol. 44, p. 94-101, D0l:10.1016/j. medengphy.2017.02.012.
[11] Gao, F., Carlton, W., Kapp, S. (2011). Effects of joint alignment and type on mechanical properties of thermoplastic articulated ankle-foot orthosis. Prosthetics and Orthotics International, vol. 35, no. 2, p. 181-189, D0l:10.1177/0309364611409617.

[12] Ramsey, J.A. (2011). Development of a method for fabricating polypropylene non-articulated dorsiflexion assist ankle foot orthoses with predetermined stiffness. Prosthetics and Orthotics International, vol. 35, no. 1, p. 54-69, DOl:10.1177/0309364610394477.

[13] Bowers, R. (2004). Non-articulated ankle-foot orthoses. Condie, E., Campbell, J., Martina, J. (eds.). Report of a Consensus Conference on the Orthotic Management of Stroke Patients, ISPO, Copenhagen, p. 87-94.

[14] Arch, E.S., Stanhope, S.J. (2015). Passive-dynamic ankle-foot orthoses substitute for ankle strength while causing adaptive gait strategies: a feasibility study. Annals of Biomedical Engineering, vol. 43, no. 2, p. 442-450, Dol:10.1007/s10439014-1067-8.

[15] Lai, H.-J., Yu, C.-H., Kao, H.-C., Chen, W.-C., Chou, C.-W., Cheng, C.-K. (2010). Ankle-foot simulator development for testing ankle-foot orthoses. Medical Engineering \& Physics, vol. 32, no. 6, p. 623-629, D0l:10.1016/j.medengphy.2010.03.008.

[16] Harvey, R.L., Macko, R.F., Stein, J., Winstein, C.J., Zorowitz, R.D. (2008). Stroke Recovery and Rehabilitation. Demos Medical Publishing, New York.

[17] Bielby, S.A., Warrick, T.J., Benson, D., Brooks, R.E., Skewes, E., Alvarez, E., Dunning, C., Desjardins, J.D. (2010). Trimline severity significantly affects rotational stiffness of ankle-foot orthosis. Journal of Prosthetics and Orthotics, vol. 22, no. 4, p. 204-210, D0I:10.1097/JP0.0b013e3181f9082e.

[18] Sumiya, T., Suzuki, Y., Kasahara, T. (1996). Stiffness control in posterior-type plastic ankle-foot orthoses: effect of ankle trimline. Part 1: A device for measuring ankle moment. Prosthetics and Orthotics International, vol. 20, no.2, p. 129131, DOI:10.3109/03093649609164430.

[19] Chu, T.-M., Reddy, N.P., Padovan, J. (1995). Three-dimensional finite element stress analysis of the polypropylene, ankle-foot orthosis: static analysis. Medical Engineering \& Physics, vol. 17, no. 5, p. 372-379, DOl:10.1016/1350-4533(95)97317-I.

[20] Chandrupatla, T. (2003). Finite Element Analysis for Engineering \& Technology. Orient Blackswan, India.

[21] Wilson, A.B. (1974). Vacuum forming of plastics in prosthetics and orthotics. Orthotics and Prosthetics, vol. 28, no. 1, p. 1220.

[22] Breuckmann, B. (2014). 25 years of high definition 3D scanning: history, state of the art, outlook. Kia, Ng., McDaid S., Bowen J. (eds.), Electronic Visualisation and the Arts, London, p. 262-266, DOI:10.14236/ewic/eva2014.31.

[23] Brajlih, T., Tasic, T., Drstvensek, I., Valentan, B., Hadzistevic, M., Pogacar, V., Balic, J., Acko, B. (2011). Possibilities of using three-dimensional optical scanning in complex geometrical inspection. Strojniški vestnik - Journal of Mechanical Engineering, vol. 57, no. 11, p. 826-833, D0l:10.5545/svjme.2010.152.

[24] Mandić, M., Galeta, T., Raos, P., Jugović, V. (2016). Dimensional accuracy of camera casing models 3D printed on Mcor IRIS: A case study. Advances in Production Engineering 
\& Management, vol. 11, no. 4, p. 324-332, Dol:10.14743/ apem2016.4.230.

[25] Jezeršek, M., Babnik, A., Možina, J., Novak, B. (2014). Threedimensional foot scanning system with a rotational laser-based measuring head. Strojniški vestnik - Journal of Mechanical Engineering, vol. 60, no. 11, p. 685-693, D0l:10.5545/svjme.2014.1950.

[26] Alam, M., Choudhury, I.A., Mamat, A.B., Hussain, S. (2015). Computer aided design and fabrication of a custom articulated ankle foot orthosis. Journal of Mechanics in Medicine and Biology, vol. 15, no. 4, DOl:10.1142/S021951941550058X.

[27] Gomes, G., Lourenço, I., Oliveira, J., Gomes, M., Vale, A., Freire, L., Quental, P., Policarpo, H., Matos, J. (2017). Structural reinforcements on AFO's: A study using computer-aided design and finite element method. IEEE $5^{\text {th }}$ Portuguese Meeting on Bioengineering, p. 77-80, D0l:10.1109/ ENBENG.2017.7889432.

[28] Munguia, J., Dalgarno, K.W. (2013). Ankle foot orthotics optimization by means of composite reinforcement of freeform structures. 24th International Solid Freeform Fabrication Symposium - An Additive Manufacturing Conference, Austin, p. $766-776$.

[29] Surmen, H.K., Akalan, N.E., Arslan, Y.Z. (2018). Design, manufacture and selection of ankle-foot-orthoses. Mehdi K.P. (ed.), Encyclopedia of Information Science and Technology, IGI Global Publications, Hershey, vol. 10, p. 298-313, DOI:10.4018/978-1-5225-2255-3. 